Jpn. J. Phys. Fitness Sports Med. 2006, 55 Suppl. : S49 \$ \$52

\title{
BODY COMPOSITION EVALUATED BY SEGMENTAL BIOELECTRICAL IMPEDANCE ANALYSIS IN HEALTHY SUBJECTS AND ATHLETES
}

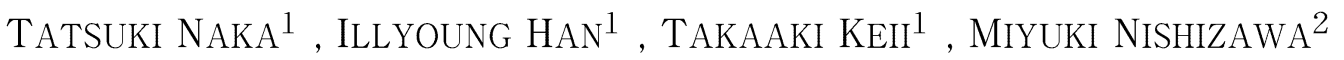 \\ Hitoshi $\mathrm{SATO}^{2)}$ and MAKOTO OHNO ${ }^{1)}$
}

\begin{abstract}
The purpose of this study was to evaluate the fat-free mass (FFM) of the total body, the upper limbs, and the lower limbs in healthy subjects and basketball players obtained by the new 8-electrode segmental bioelectrical impedance analysis (S-BIA) and to compare the results with the FFM obtained by DXA. The participants were 30 healthy subjects ( 15 men and 15 women) aged $24.9 \pm 3.5$ years old and 15 female basketball players aged $23.1 \pm 3.1$ years old. In the healthy subjects, positive correlations $(\mathrm{p}<0.001)$ were observed between the FFM obtained by S-BIA and DXA of the total body $(r=0.973)$, the upper limbs $(r=0.956)$, and the lower limbs $(r=0.954)$. Similarly, in the basketball players, positive correlations $(\mathrm{p}<0.001, \mathrm{p}<0.01)$ were observed between the FFM obtained by S-BIA and DXA of the total body $(r=0.943)$, the upper limbs $(r=$ $0.743)$, and the lower limbs $(r=0.934)$. The results suggest that the new 8-electrode S-BIA is a valid and convenient method for analyzing body composition of the total body and the body segments in healthy subjects and athletes.
\end{abstract}

(Jpn. J. Phys. Fitness Sports Med. 2006, 55 Suppl : S49 S52)

key word : body composition, S-BIA, DXA, healthy subject, basketball player

\section{Introduction}

Body composition is not only one of the factors to affect sports/exercise related performance but also one of the indices of physical conditioning. Total body composition analys is is useful to prevent life-style related diseases such as obesity, to evaluate nutritional status in ordinary people. Nowadays, body composition analysis in the body segments is considered much more important than total body composition analysis in the athlete as well as in ordinary people.

However, many previous studies ${ }^{1,2)}$ have focused on total body composition rather than on the segmental body composition.

Recently, segmental body composition in the many parts of the human body can be analyzed precisely using the whole body scan with dual energy $\mathrm{X}$-ray absorptiometry (DXA). Many studies ${ }^{3 \sim 5}$ ) have confirmed that DXA is a precise and valid form of body composition analysis.

However, the DXA machine was large and could not be moved. An instrument that could measure in a short time and be used spused anywhere was eagerly needed for investigation of a large number of subjects, effective judgment for conditioning and training, and chronological observation of daily life. Thus, bioelectrical impedance analysis (BIA) which is a simple body composition evaluation method was devised ${ }^{6 \sim 8)}$, but BIA can analyze only the total body composition. Finally, the new 8-electrode segmental BIA (S-BIA) was recently developed for measuring the segmental body composition and evaluating the body composition easily.

The purpose of this study was to evaluate the fat-free mass (FFM) of the total body, the upper limbs, and the lower limbs in healthy subjects and basketball players obtained by the new 8-electrode S-BIA and to compare the results with the FFM obtained by DXA.

\section{Methods}

\section{Subjects}

The participants were 30 healthy subjects (men/

\footnotetext{
1) Graduate School of Health and Sport Science, Nippon Sport Science University, Japan

${ }^{2)}$ Research and Development Department, Tanita Corporation, Japan
} 
women $=15 / 15$, age $: 24.9 \pm 3.5$ y/o, BMI $: 22.8 \pm$ $2.4 \mathrm{~kg} / \mathrm{m}^{2}$ ) and 15 female basketball players (age : $23.1 \pm 3.1 \mathrm{y} / \mathrm{o}$, BMI $: 21.8 \pm 1.3 \mathrm{~kg} / \mathrm{m}^{2}$ ). The healthy subjects were college students who had not exercised for 1 year or more. The basketball players were the top players belonging to Women's Japan Basketball League Organization (WJBL).

2. Body composition analysis (FFM) by $S-B I A$

We analyzed body composition (FFM) using the new 8-electrode S-BIA (TANITA, BC-118 modify). First all subjects stand on this instrument and grasp two handgrips. Then the segmental body composition is measured using impedance collected by an electric current through both hands and both feet. We are also able to measure body segmental impedance by changing the electrode point and voltage to send an electric current using the peculiar new 8-electrode method.

Measurement segments were the total body, the upper limbs, and the lower limbs. The time needed for the measurement was approximately 1 minute per person. All subjects were measured at the same time (noon) after urination.

The conditions for the measurement of body composition were as follows :

1) The subjects did not carry out drastic exercise 12 hours prior to the measurement.

2) The subjects got up at least three hours before the measurement and had breakfast, and did not eat or drink anything after breakfast.

3) The subjects performed activities of daily living and did not remain in a sitting position or recumbency for an extended period after getting up.

3. Body composition analysis (FFM) by DXA

We analyzed body composition (FFM) using DXA (LUNAR, DPX). Measurement segments were the total body, the upper limbs, and the lower limbs. The time needed for the measurement was approximately 15 minutes per person.

\section{Results}

In the healthy subjects, positive correlations $(\mathrm{p}<$ 0.001 ) were observed between the FFM of the total body obtained by S-BIA and DXA ( $y=0.963 \mathrm{x}+$ $0.40, r=0.973$, Fig. 1). Similarly, positive correlations $(p<0.001$ ) were observed between the FFM of the upper limbs obtained by S-BIA and DXA ( $y=$ $0.900 x+0.33, r=0.956$, Fig. 2). In addition, positive correlations $(\mathrm{p}<0.001)$ were observed between the FFM of the lower limbs obtained by S-BIA and DXA $(y=0.894 x+1.10, r=0.954$, Fig. 3$)$.

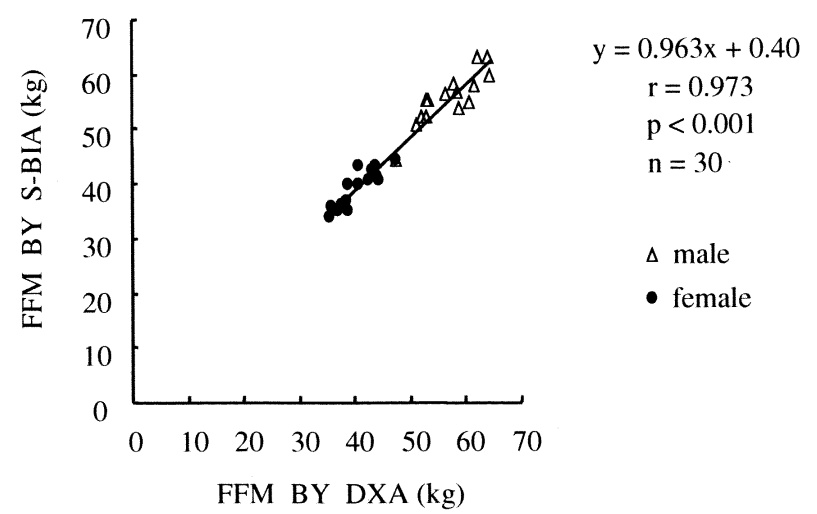

Fig. 1. Relationship between the FFM obtained by SBIA and DXA of the total body in healthy subjects.

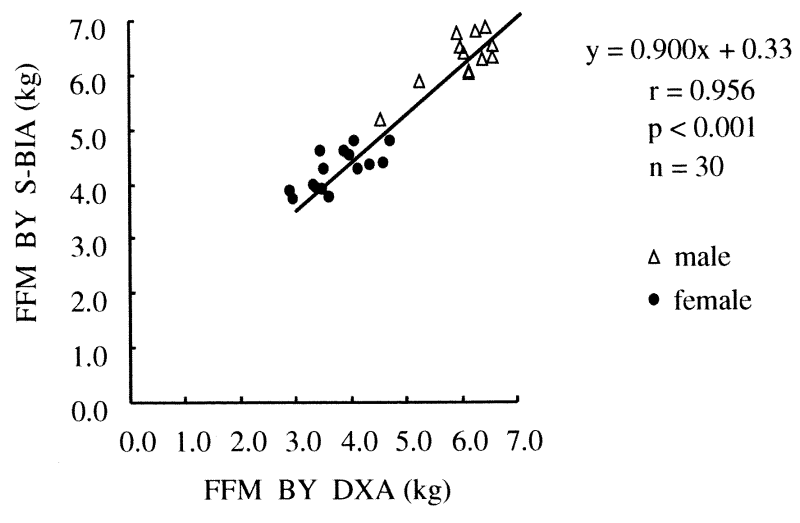

Fig. 2. Relationship between the FFM obtained by SBIA and DXA of the upper limbs in healthy subjects.

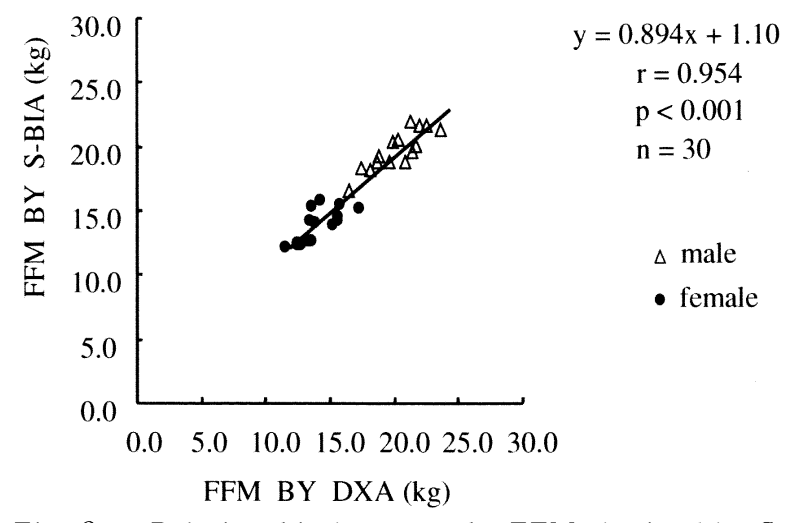

Fig. 3. Relationship between the FFM obtained by SBIA and DXA of the lower limbs in healthy subjects. 


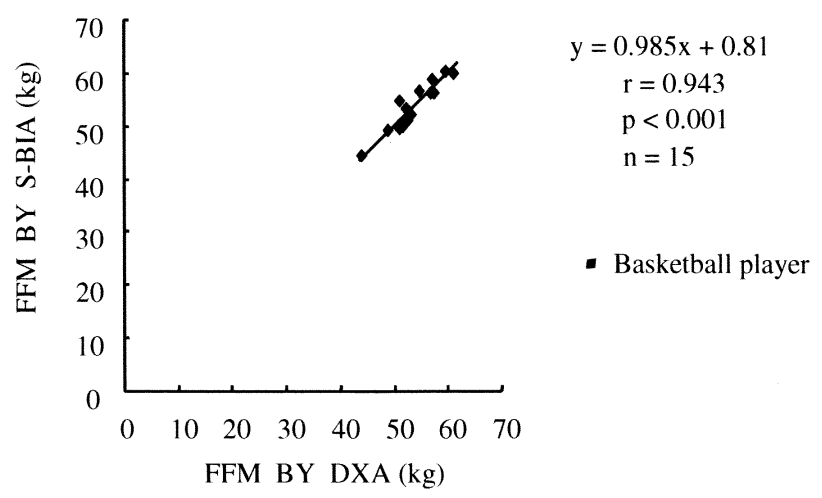

Fig. 4. Relationship between the FFM obtained by SBIA and DXA of the total body in basketball players.

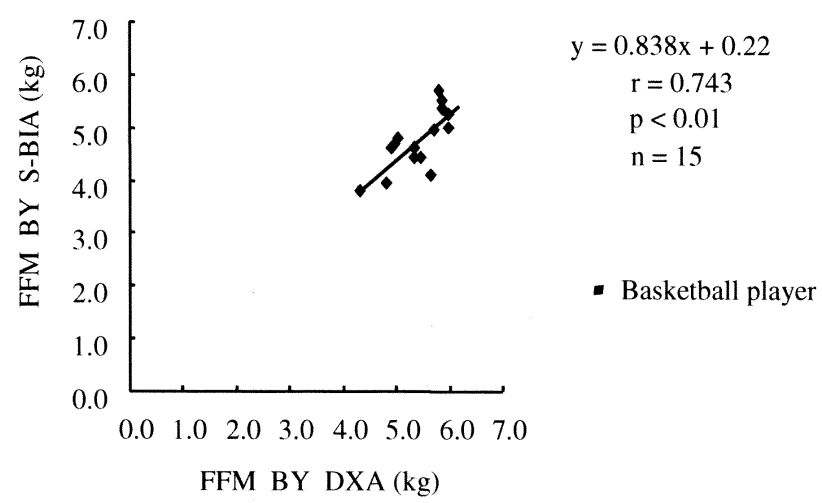

Fig. 5. Relationship between the FFM obtained by SBIA and DXA of the lower limbs in basketball players.

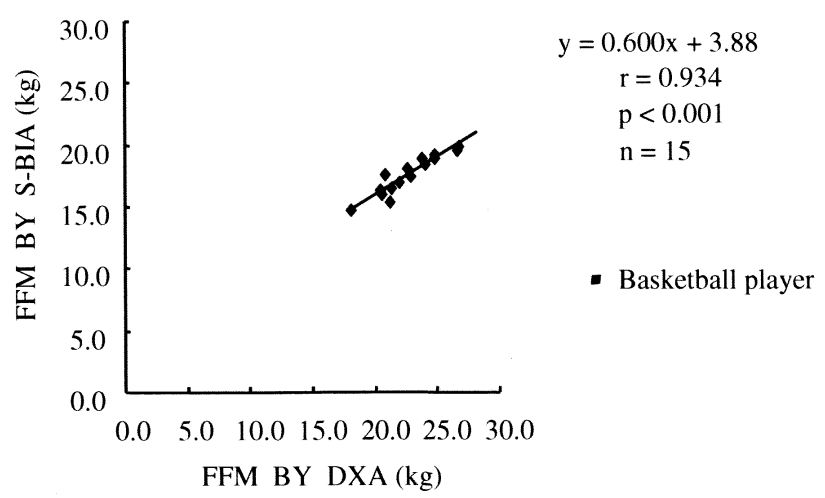

Fig. 6. Relationship between the FFM obtained by SBIA and DXA of the lower limbs in basketball players.

In the basketball players, positive correlations ( $\mathrm{p}$ $<0.001$ ) were observed between the FFM of the total body obtained by S-BIA and DXA $(y=0.985 x+$ $0.81, r=0.943$, Fig. 4). Similarly, positive correlations $(p<0.01$ ) were observed between the FFM of the upper limbs obtained by S-BIA and DXA ( $y=$ $0.838 \mathrm{x}+0.22, \mathrm{r}=0.743$, Fig. 5 ). In addition, positive correlations $(p<0.001)$ were observed between the FFM of the lower limbs obtained by S-BIA and DXA $(y=0.600 x+3.88, r=0.934$, Fig. 6$)$.

\section{Discussion}

In the healthy subjects, positive correlations were observed between the FFM of the total body obtained by the 8-electrode S-BIA and DXA. Similarly, positive correlations were observed between the FFM of the upper limbs and the lower limbs obtained by the 8-electrode S-BIA and DXA. There were no differences between the data in the total body, the upper limbs, and the lower limbs obtained by the 8 -electrode S-BIA and DXA. Thus, it seems to be clear that the 8-electrode S-BIA can analyze body composition of the total body and the body segments (the upper limbs and the lower limbs) with the same precision as DXA. Algorithmic validity of this instrument was confirmed.

In the basketball players, positive correlations were observed between the FFM of the total body obtained by the 8-electrode S-BIA and DXA. Similarly, Positive correlations were observed between the FFM of the upper limbs obtained by the 8electrode S-BIA and DXA. There were no the differences between the data in the total body and the upper limbs obtained by the 8-electrode S-BIA and DXA. Even though positive correlations were also observed between the FFM in the lower limbs obtained by the 8-electrode S-BIA and DXA, the regression coefficient was low. Namely, the FFM in the lower limbs obtained by the 8-electrode S-BIA was lower than that by DXA.

We believe that the reason this happened was due to the fact that the cross section of the lower limbs is more massive than that of the upper limbs. The impedance of the lower limbs is small, so that the values of the FFM of the lower limbs tend to be more uneven than that of the upper limbs. The basketball players have a larger volume of muscle mass than the healthy subjects. Thus, the regression coefficient of the basketball players was lower than in the healthy subjects. We intend to investigate this problem in detail with as many subjects as possible 
in further studies.

Thus, it seems to be clear that the 8-electrode S-BIA can analyze body composition of the total body and body segments (the upper limbs and the lower limbs) for not only healthy subjects but also athletes, with the same precision as DXA.

But there is a circadian rhythm impedance. The impedance is affected by changes of quantity and distribution of total body water and variations of the conductivity by body temperature ${ }^{9,10)}$. Therefore, if S-BIA is carefully measured in a constant condition on the basis of matters that require attention in the measurement, the 8-electrode S-BIA is a valid and convenient method for both healthy subjects and athletes to analyze the total and segmental body composition, with the same high precision as DXA. The new 8-electrode S-BIA is considered to be very useful to clarify physical characteristics of many numbers of athletes in many kinds of sports.

\section{Referrences}

1) Tahara, Y., Tsunawake, N., Michimukou, R., Senjyu, H., Yukawa, K. Sports woman no shintaisosei no syumokutokusei. Orthop. Surg. Traumatol.,(1993), 36, 1165-1170. (in Japanese)

2) Takenoshita, H. \&, Taimura, A. A Study on the Change of Body Composition in College Female Bas- ketball Players. J. Meth. Sports,(1989), 2, 32-42.

3) Mazess, R. B., Barden, H. S., Bisek, J. P., Hanson, J. Dual-energy $\mathrm{x}$-ray absorptiometry for total-body and regional bone-mineral and soft-tissue composition. Am. J. Clin. Nutr.,(1990), 51, 1106-1112.

4) Lukaski, H. C. Methods for the assessment of human body composition : traditional and new. Am. J. Clin. Nutr.,(1987), 46, 537-556.

5) Ramsdale, S. J. \&, Bassey, E. J. Changes in bone mineral density associated with dietary-induced loss of body mass in young women. Clin. Sci.,(1994), 87, 343-348.

6) Houtkooper, L. B., Going, S. B., Lohman, T. G., Roche, A. F., Van Loan, M. Bioelectrical impedance estimation of fat-free body mass in children and youth : a cross-validation study. J. Appl. Physiol.,(1992), 72, 366-373.

7) Van Marken Lichtenbelt, W. D., Westerterp, K. R., Wouters, L., Luijendijk, S. C. Validation of bioelectrical-impedance measurements as a method to estimate body-water compartments. Am. J. Clin. Nutr., (1994), 60, 159-166.

8) Nakadomo, F., Tanaka, K., Hazawa, T., Maeda, K. Validation of body composition assessed by bioelectrical impedance analysis. Jpn. J. Appl. Physiol., (1990), 20, 321-330.

9) Sakamoto, Y., Sato, H., Ikeda, Y. Taishiboryo no sokuteiho. Jpn. J. clin. med.,(2003), 61, 368-373. (in Japanese)

$10)$ Sato, H. \&, Sakamoto, Y. Taishibo to sokuteiho. Diagnosis and treatment, (1996), 84, 975-979. (in Japanese) 